\title{
The association between obesity and migraine and possible mechanisms of action: an integrative literature review
}

\author{
Dieniffer Aparecida Halaiko1 (iD) Paulo Faro² (iD) Aline Andretta Levis ${ }^{3}$ (iD) Bárbara Dal Molin Netto3 (iD \\ ${ }^{1}$ Federal University of Paraná, Curitiba, Paraná, Brazil \\ ${ }^{2}$ Institute of Neurology of Curitiba - INC, Curitiba, Paraná, Brazil \\ ${ }^{3}$ Federal University of Paraná, Curitiba, Paraná, Brazil
}

Dieniffer Aparecida Halaiko
Email: dienifferhalaiko@gmail.com Bárbara Dal Molin Netto

Email: barbaradm@ufpr.br

\section{Edited by}

Mario Fernando Prieto Peres Marcelo Moraes Valença

\section{Keywords:}

Migraine Disorders

Obesity

Inflammation

Adipose Tissue

Risk Factors

Neuropeptides

\begin{abstract}
Introduction

Obesity is a multifactorial disease defined by the excessive accumulation of adipose tissue that can cause harm to human health. The presence of obesity is an important risk factor for migraine chronification. However, not much is known about the link between the two diseases.

\section{Methods}

In this study, an integrative literature review was conducted to better understand the mechanisms of interaction between migraine and obesity. Therefore, a search of PubMed and the Virtual Health Library ( $\mathrm{VHL}$ ) was performed with the following keywords: enxaqueca e obesidade; enxaqueca e obesidade e inflamação; enxaqueca e obesidade e neuropeptídeos; migraine and obesity; migraine and obesity and inflammation; migraine and obesity and neuropeptides.

\section{Results}

The search identified 22 articles. After reading and analyzing the articles, three thematic categories emerged: 1) Obesity as an Aggravating Factor for Migraine, 2) Mechanisms Studied between Obesity and Migraine and 3) The Effect of Weight Loss on Migraine Symptoms.

\section{Conclusions}

The chronic low-grade inflammation associated with obesity can cause a predisposition to migraine chronification. The abnormal secretion of adipokines, dysregulation of the sympathetic nervous system, and hypothalamic dysfunction have been suggested to be the main shared mechanisms between both diseases.
\end{abstract}




\section{Introduction}

( ) besity is a chronic disease defined by the excessive accumulation of adipose tissue, resulting from an energy imbalance, which can cause damage to human health. 'The worldwide prevalence of obesity practically tripled between the years 1975 and 2016, and in 2016 more than 1.9 billion adults were overweight, of which over 650 million were obese. Globally, there are more obese than underweight individuals. ${ }^{2}$ In Brazil, the prevalence of overweight is $60.3 \%$, affecting 96 million individuals, and one third of men and a half of women are obese. ${ }^{3}$

The etiology of obesity is multifactorial and involves genetic, behavioral, socioeconomic and cultural factors. ${ }^{4}$ The inadequate interaction between the aforementioned factors may favor an imbalance in energy balance, which promotes lipogenesis and, consequently, body weight gain. ${ }^{5}$ This accumulation of adipose tissue and increase in body mass index (BMI) increase the risk of developing chronic non-communicable diseases, such as systemic arterial hypertension, dyslipidemia, cardiovascular disease, diabetes mellitus, osteoarthrosis, obstructive sleep apnea syndrome, some types of cancer ${ }^{6}$ and even migraine. ${ }^{7,8}$ In addition, obesity can also lead to mental illnesses, such as depression. ${ }^{9}$

Migraine is considered a common primary headache, and is classified as the sixth most prevalent disorder worldwide and as the first cause of disability among men and women under 50 years old. ${ }^{10,11}$

It is known that the etiology of migraine is genetic ${ }^{12}$ and that obesity is an important risk factor for the worsening of its symptoms. There is strong evidence showing an increased risk of developing migraine in $50 \%$ of individuals with obesity ${ }^{7}$, and migraine attacks appear to be associated with higher BMls. $^{13}$

Obesity also seems to interfere with the frequency ${ }^{14,15}$, duration and intensity of migraine symptoms ${ }^{16}$, in addition, it can increase the susceptibility of migraine to chronicity. ${ }^{17,18}$ Recent studies prove this relationship and suggest some mechanisms for the interaction between the two diseases, such as inflammation $^{19}$, persistent states of hyperleptinemia ${ }^{20}$, irregular hypothalamic function and sympathetic dysregulation. ${ }^{21}$

Considering the above, the scientific literature was reviewed in order to better understand the mechanisms of interaction between obesity and migraine, in addition to their clinical implications and the consequences for the health of the individuals affected by both diseases.

\section{Methods}

The present study is an integrative literature review, which includes different types of studies (experimental, non-experimental and reviews), since a broad understanding of the phenomenon under study is sought.

To this end, the following steps were taken to develop the integrative review: the definition of the problem to be researched, a search of the literature (the selection of keywords, outlining the criteria for selecting articles, and definition of the databases). The search for articles was carried out in the PubMed and Virtual Health Library (VHL) databases and the inclusion criteria adopted were articles published between 2015 and 2020, written in Portuguese or English and that presented in their discussions considerations about the role of obesity in migraine. This search took place between the months of August and September 2020.

To perform the search, the following keywords were used, considered as descriptors DeCS (Descriptors in Health Sciences), in Portuguese, and MeSH (Medical Subject Headings), in English: enxaqueca e obesidade; enxaqueca e obesidade e inflamação; enxaqueca e obesidade e neuropeptídeos; migraine and obesity; migraine and obesity and inflammation; migraine and obesity and neuropeptides.

The total number of articles found using the search terms described was 54 in the Virtual Health Library (VHL) and 57 in PubMed. The selection of articles for analysis was performed through an exploratory reading of the title of all articles found in each of the databases and summary of 45 articles, and then 24 were selected for full reading. After the analytical reading, 22 articles were selected that met the criteria and answered the guiding question of this review. The steps taken to develop the integrative review are described in Table 1.

\section{Results and Discussion}

After reading and analyzing the selected articles, they were divided into three categories according to their approaches: 1) Obesity as an aggravating factor for migraine; 2) Mechanisms studied between obesity and migraine; and 3) The effect of weight loss on migraine symptoms.

\section{Obesity as an Aggravating Factor for Migraine}

The review found a significant amount of evidence showing that there is a relationship between the diseases. The research showed that overweight and obesity were considered risk 
Table 1. Description of the completed steps for the development of the integrative review

\begin{tabular}{|c|c|c|c|c|c|c|}
\hline Data base & Keywords & $\begin{array}{l}\text { Number of references } \\
\text { obtained }\end{array}$ & $\begin{array}{l}\text { References } \\
\text { sorted by tifle }\end{array}$ & $\begin{array}{l}\text { Abstracts } \\
\text { analyzed }\end{array}$ & $\begin{array}{l}\text { References selected } \\
\text { for analysis }\end{array}$ & Total for review \\
\hline \multirow[t]{8}{*}{ PubMed } & Obesity/ & 48 & 29 & 19 & 13 & 13 \\
\hline & Migraine & & & & & \\
\hline & Obesity/ & 6 & 2 & 4 & 0 & 0 \\
\hline & Migraine/ & & & & & \\
\hline & Inflammation & & & & & \\
\hline & Obesity/ & 3 & 3 & 0 & 0 & 0 \\
\hline & Migraine/ & & & & & \\
\hline & Neuropeptides & & & & & \\
\hline \multirow[t]{8}{*}{$\mathrm{VHL}$} & Obesity/ & 54 & 32 & 22 & 11 & 9 \\
\hline & Migraine & & & & & \\
\hline & Obesity/ & 0 & 0 & 0 & 0 & 0 \\
\hline & Migraine/ & & & & & \\
\hline & Inflammation & & & & & \\
\hline & Obesity/ & 0 & 0 & 0 & 0 & 0 \\
\hline & $\begin{array}{l}\text { Migraine/des } \\
\text { Neuropeptides }\end{array}$ & & & & & \\
\hline & TOTAL & 111 & 66 & 45 & 24 & 22 \\
\hline
\end{tabular}

factors for migraine chronicity ${ }^{18}$ and that obesity can increase the risk of migraine by up to $50 \%$, a risk that increases with the increase in the degree of obesity. ${ }^{7}$ Moreover, the evidence suggests that the chance of having a daily migraine crisis is associated with a higher BMI. ${ }^{13}$ In addition, Miri et al. ${ }^{22}$ found a positive association between individuals with obesity and the presence of migraine with aura. Obese individuals were 2.92 times more likely to have migraine with aura than individuals with normal weight. ${ }^{22}$ Corroborating these results, Huang et al. ${ }^{14}$ found that the higher the frequency of episodic migraine attacks, the greater the $\mathrm{BMI}$, but this association was not observed between the severity and duration of headache, nor in individuals with chronic migraine. ${ }^{14}$

Although there appears to be a relationship between obesity and the chronicity of migraine, several other risk factors possibly influence the relationship. ${ }^{23}$ Increased adiposity and the consequent low-grade chronic inflammation can interfere with the natural history of migraine ${ }^{24}$, and there is a clear relationship between obesity and migraine; however, a number of other risk factors can also have a possible influence on migraine ${ }^{23}$ including genetic predisposition, age and sex. ${ }^{8}$

In respect of sex, for example, women with obesity are more likely to have migraine when compared to men ${ }^{8,18,25}$, especially women of reproductive age. ${ }^{7}$ The increased risk of migraine in women with obesity can be explained by changes in sex hormones and low-grade inflammation due to the higher percentage of body fat present in obesity. ${ }^{26}$ Furthermore, an experimental study carried out with rats ${ }^{26}$ showed that female rats are more sensitive to light, and the presence of obesity was shown to aggravate this symptom. Another important factor in respect of this relationship is that women with migraine and obesity tend to be less active than obese women without migraine. The study by Bond et al. ${ }^{27}$ concluded that women with obesity and migraine practiced 1.5 hours less daily physical activity when compared to obese women without migraine. ${ }^{27}$

There are also differences in the distribution and metabolic function of adipocytes in relation to age and sex, which may affect the relationship between obesity and migraine. ${ }^{8}$ Moreover, the duration of obesity and the distribution of body fat in women with obesity may also be influenced by variations in the hormonal concentrations of menstrual cycles during life, which in turn may have an influence on migraine. ${ }^{24}$

\section{Mechanisms Studied between Obesity and Migraine}

Studies that analyze the mechanisms by which migraine is associated with obesity are still incipient. The inflammation seen in patients with obesity may be one of the causes of the high prevalence and progression of migraine in individuals with both diseases. ${ }^{14,18,19,22,28,29}$ The expansion of adipose tissue, which happens with obesity, leads to an alteration in the synthesis of pro-inflammatory markers, favoring chronic 
inflammation and insulin resistance. ${ }^{7}$ C-reactive protein (CRP), tumor necrosis factor-a (TNF-a), interleukin-1 (IL-1), interleukin-6 (IL-6), substance $P$ and peptide related to the calcitonin gene (CGRP) were found in high concentrations in individuals with obesity and migraine, especially during crises, when compared to individuals without obesity and without migraine. 18,19,21 These inflammatory mediators can sensitize the central nervous system causing permanent damage to the periaqueductal gray matter, an area closely related to the pathophysiology of migraine and its modulation. ${ }^{16}$

In turn, CGRP, which is an important inflammatory mediator in migraine, was also found at high levels in individuals with obesity. ${ }^{14,25}$ Substance P (SP), on the other hand, seems to play a role in the accumulation of fat and in the beginning of the inflammatory cascade related to obesity, which may be associated with an increase in susceptibility to trigeminal stimulation and, consequently, leading to migraine attacks. ${ }^{19}$

It is also important to highlight the possible effects of elevated levels of proinflammatory cytokines on the trigeminovascular system involved in stimulating migraine pain. This may explain, at least partially, the more frequent and severe headaches in individuals with obesity ${ }^{21}$ and how these changes in the trigeminal nociceptive pathway cause central sensitization, which favors the evolution of the disease from its episodic to chronic form. ${ }^{17}$

Adipokines produced by adipose tissue, leptin and adiponectin, have also been shown to be related to migraine. In contrast to other adipokines, adiponectin is present in higher concentrations in individuals classified with normal BMI than in those with obesity. Changes in circulating levels of adiponectin and leptin are strictly correlated with the development of insulin resistance, inflammatory processes, obesity and regulation of adipogenesis. ${ }^{21,30,31}$ On the other hand, the literature contains conflicting results regarding the effects of changes in the serum concentrations of these adipokines on the pathophysiology of migraine..$^{18}$

It is important to note that, as pointed out in a number of the studies, adiponectin levels were found to be altered in individuals both during crises and in the intercritical phase. ${ }^{8}$ One study found that this adipokine appears to be below normal levels during migraine attacks. It is assumed that low levels of adiponectin may activate the production of TNF-a, modulating the functions and phenotype of macrophages, increasing inflammation and nociceptive stimulation..$^{19}$ In addition, other studies indicate that this adipokine can act to worsen migraine by activating the nitric oxide pathway and inducing a pro-inflammatory state. ${ }^{18}$
It should be noted that research on leptin in individuals with migraine is not yet conclusive. There are assumptions about a negative relationship between leptin and pain intensity, since individuals with migraine have lower levels of leptin during attacks and higher levels in the symptom-free phase. Leptin may be involved in migraine through several mechanisms. ${ }^{19}$ A picture of hyperleptinemia seems to increase the susceptibility to cortical spreading depression, since high levels of leptin cause inhibition in the production and release of serotonin and orexin- $\mathrm{A}$, hormones that when at low levels increase the frequency of cortical spreading depression. ${ }^{20}$ Increased levels of leptin can also induce the secretion of pro-inflammatory cytokines, regulating immunoreaction and inflammatory reaction, such as IL-6 and TNF-a, arachidonic acids and nitric oxide (NO) through the nuclear factor kappa $B\left(N F_{K} B\right)$ signaling pathway. ${ }^{16,19}$ In contrast, Ligong et al. ${ }^{16}$ found no relationship between changes in leptin levels in individuals with migraine.

The interaction between obesity and migraine also seems to be shared by a bidirectional action involving hypothalamic dysfunction ${ }^{16,19,20,28}$, since obesity results from a dysregulation in energy metabolism and these changes have been associated with migraine. ${ }^{29}$ This hypothesis was reinforced by the performance of imaging tests, which revealed greater hypothalamic activation, responsible for the control of hunger, in the onset of migraine crises. ${ }^{7}$ This may be due to over-activation of the reward system, obesity linked to overeating behaviors and migraine to overuse of medicines. ${ }^{28}$ It is also noteworthy that the neuropeptides and neurotransmitters (serotonin, adiponectin, leptin and orexin) that are involved in the control of the energy balance, also seem to be involved in the pathophysiology of migraine ${ }^{7,28,32}$. The increase observed in the concentration of serotonin secreted by platelets during crises may be one of the causes of vasoconstriction of arteries that are part of the phenomenon of cortical spreading depression. ${ }^{19}$

Another possible mechanism is attributed to levels of orexin-A, as low concentrations were found in women with obesity, and it is assumed that the deficiency of this substance promotes inflammation of the trigeminal system contributing to the development of migraine. ${ }^{21}$ High levels of orexin were observed in the cerebrospinal fluid of individuals with migraine and was attributed to compensatory reactions capable of increasing pain. The literature also suggests that dysfunctions in orexigenic mechanisms can lead to increased appetite and be related to homeostatic pathways that are involved in the risk of generating the premonitory phase and migraine headache.19 
Recent evidence indicates that dysregulation of the sympathetic nervous system may be one of the links between obesity and increased seizures in migraine patients. It is known that individuals with obesity are often resistant to the action of leptin in the central nervous system, which can contribute to an increase in the risk of developing sympathetic hyperactivity. High sympathetic tone is a common feature of obesity, which can lead to a higher frequency of headache. ${ }^{21}$

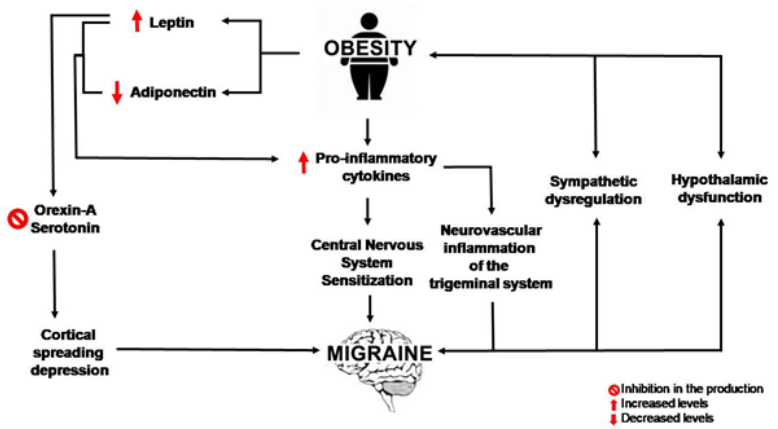

Figure 1. Shared mechanisms between obesity and migraine

\section{The Effect of Weight Loss on Migraine Symptoms}

In view of the strong evidence in the literature on the impact of obesity on the frequency, duration and severity of migraine ${ }^{16}$, and its transformation from episodic to chronic migraine, it is important to consider approaches aimed at treating obesity in migraine patients as it is a modifiable risk factor. ${ }^{25}$

Some studies have been developed to evaluate weight loss as a treatment for migraine. The study by RazeghiJahromi et al..$^{31}$ found that the surgical treatment of obesity through gastric sleeve surgery in women with migraine promoted a reduction in the severity and duration of crises, in addition to providing a greater number of days without migraine. ${ }^{31}$ Corroborating these findings, a systematic review study with bariatric patients observed a reduction in migraine frequen$c y$, headache-related disability and pain intensity 6 months after surgery. ${ }^{21}$

The improvement in migraine can be explained by the effects of weight loss on metabolic abnormalities, decreased circulating levels of inflammatory adipokines related to migraine, and decreased hormones produced by adipocytes due to a reduction in the size of this cell. ${ }^{21}$ Studies have shown that gastric surgery can produce a significant decrease in the levels of glucose, insulin, leptin and adrenocorticotrophic hormone $(\mathrm{ACTH})$ one month after surgery. ${ }^{31}$

Reinforcing the influence of obesity on migraine, Di Vincenzo et al. ${ }^{29}$ compared surgical and non-surgical weight loss as treatment methods for migraine, and reported positive results, with similar benefits found in respect of the frequency, intensity of pain, disability and duration of crisis, regardless of the type of weight-loss treatment. ${ }^{29}$ Bond et al. ${ }^{15}$ sought to evaluate the effects of two intervention methods on migraine, conventional weight loss and a migraine control educational protocol. Similar improvements in the frequency of migraine were observed in respect of both interventions. The authors suggest that the effects of conventional weight loss on migraine are due to improvements in physiological, psychological and behavioral factors, such as inflammation, depression and physical activity, respectively. In respect of the migraine education, the benefits can be attributed to greater knowledge of the causes, triggers and behaviors related to crises, as well as the correct way to manage them. ${ }^{15}$

The mechanisms by which weight loss acts to control migraine in individuals with obesity are not yet fully understood, as studies have shown a reduction in the frequency of crises, the intensity of the migraine and related disability even before any significant weight loss; it has been suggested that even the small changes in the size of adipocytes following weight loss programs can be beneficial in respect of the release of adipokines observed before any major changes in weight. The negative energy balance resulting from different strategies for weight reduction seems to generate a decrease in circulating pro-inflammatory cytokines and an increase in anti-inflammatory cytokines, which may have a positive impact on the control of migraine crises. ${ }^{29}$ Thus, the literature indicates that normalizing adipokine levels through weight loss can improve the pro-inflammatory state responsible for increasing the frequency and intensity of headache. ${ }^{21}$

Several characteristics are shared between both diseases and are involved in their triggering and/or worsening, such as: depression, anxiety, fluctuations in stress, stressful events in childhood, short sleep duration, irregular meals, more Westernized diets (rich in fats and sugars combined), a sedentary lifestyle, as well as prolonged fasting and the use of certain medications. ${ }^{8,28}$ Moreover, the atherogenic profile observed in individuals with migraine shares common features with the profile of individuals with obesity, and is possibly related to a higher incidence of migraine in individuals with obesity. ${ }^{21}$

\section{Limitations}

Considering the studies evaluated in the present review on the relationship between obesity and migraine, there are some factors that can limit the expansion of knowledge about the association of these disorders, such as: 
a) Sample size and selectivity: since many of the studies comprise samples of women of reproductive age who have undergone weight loss, the results cannot be generalized to other populations;

b) Absence of control groups with individuals with normal weight and migraine;

c) Lack of differentiation between migraine and other types of headache;

d) Use of migraine drugs in research aimed at evaluating the symptoms of headache after weight loss;

e) Not following standard classifications for migraine and BMI. In addition, in order to have greater validity in respect of the nutritional diagnosis, it is necessary to use methods that assess body composition and levels of adipose tissue.

\section{Conclusions}

We conclude that the chronic low-grade inflammation associated with obesity may predispose individuals to migraine, in addition to increasing the number and severity of migraines and the associated disability. Furthermore, abnormal adipokine secretion, sympathetic dysregulation and hypothalamic dysfunction have been suggested as mechanisms shared between obesity and migraine. It should also be noted that it is well established in the literature that weight loss can decrease the frequency, intensity and duration of crises, as well as migraine-related incapacity.

Conflict Of Interest: There is no conflict of interest.

Author contributions: BDMN, DAH and AAL - Article planning, definition of research strategies and guiding question, critical review of intellectual content; BDMN, DAH and PF - Final approval of the version to be published.

Dieniffer Aparecida Halaiko

https://orcid.org/0000-0003-1074-7936

Paulo Faro

https://orcid.org/0000-0001-8932-4164

Aline Andretta Levis

https://orcid.org/0000-0001-7578-2375

Bárbara Dal Molin Netto

https://orcid.org/0000-0003-0748-7141

\section{References}

1. World Health Organization (WHO). Obesity: preventing and managing the global epidemic: report of a WHO consultation. Geneva: World Health Organization; 2000. 252p. WHO Technical Report Series, 894.

2. World Health Organization (WHO). Obesity and overweight. 2021 [Available from: https://www.who.int/news-room/ fact-sheets/detail/obesity-and-overweight.]

3. Intituto Brasileiro de Geografia e Estatística (IBGE). Atenção primária à saúde e informações antropométricas. Pesquisa Nacional de Saúde. 2019. p. 1-66.

4. Thaker VV. Genetic and epigenetic causes of obesity. Adolesc Med State Art Rev 2017;28(2):379-405

5. Ferreira APS, Szwarcwald CL and Damacena GN. Prevalência e fatores associados da obesidade na população brasileira: estudo com dados aferidos da Pesquisa Nacional de Saúde, 2013. Revista Brasileira de Epidemiologia 2019;22. Doi: 10.1590/1980-549720190024

6. Riaz H, Khan MS, Siddiqi TJ, Usman MS, Shah N, Goyal A, ... Ahmed H. Association Between Obesity and Cardiovascular Outcomes: A Systematic Review and Meta-analysis of Mendelian Randomization Studies. JAMA Netw Open 2018;1(7):e183788 Doi: 10.1001/jamanetworkopen. 2018.3788

7. Klenofsky B, Pace A, Natbony LR and Sheikh HU. Episodic Migraine Comorbidities: Avoiding Piffalls and Taking Therapeutic Opportunities. Curr Pain Headache Rep 2019;23(1):1 Doi: 10.1007/s1 1916-019-0742-8

8. Gelaye B, Sacco S, Brown WJ, Nitchie HL, Ornello R and Peterlin BL. Body composition status and the risk of migraine: A meta-analysis. Neurology 2017;88(19):1795-1804 Doi: 10.1212/wnl.0000000000003919

9. Rao WW, Zong $Q Q$, Zhang JW, An FR, Jackson T, Ungvari $G S, \ldots$ Xiang YT. Obesity increases the risk of depression in children and adolescents: Results from a systematic review and meta-analysis. J Affect Disord 2020;267(78-85 Doi: 10.1016/j.jad.2020.01.154

10. Headache Classification Committee of the International Headache Society (IHS) The International Classification of Headache Disorders, 3rd edition. Cephalalgia 2018;38(1):1-21 1 Doi: 10.1177/0333102417738202

11. Global, regional, and national incidence, prevalence, and years lived with disability for 328 diseases and injuries for 195 countries, 1990-2016: a systematic analysis for the Global Burden of Disease Study 2016. Lancet 2017;390(10100): 1211. 1259 Doi: 10.1016/s0140-6736(17)32154-2

12. Gormley P, Anttila V, Winsvold BS, Palta P, Esko T, Pers TH, . . Palotie A. Meta-analysis of $\mathbf{3 7 5 , 0 0 0}$ individuals identifies $\mathbf{3 8}$ susceptibility loci for migraine. Nat Genet 2016;48(8):856866 Doi: 10.1038/ng.3598

13. Santos IS, Goulart AC, Passos VM, Molina Mdel C, Lotufo PA and Bensenor IM. Obesity, abdominal obesity and migraine: a cross-sectional analysis of ELSA-Bra- 
sil baseline data. Cephalalgia 2015;35(5):426-436 Doi: 10.1177/0333102414544978

14. Huang $Q$, Liang $X$, Wang $S$ and Mu X. Association between Body Mass Index and Migraine: A Survey of Adult Population in China. Behav Neurol 2018;2018(6585734 Doi: 10.1155/2018/6585734

15. Bond DS, Thomas JG, Lipton RB, Roth J, Pavlovic JM, Rathier L, . . Wing RR. Behavioral Weight Loss Intervention for Migraine: A Randomized Controlled Trial. Obesity (Silver Spring) 2018;26(1):81-87 Doi: 10.1002/oby.22069

16. Ligong Z, Jinjin $Q$, Chunfu $C$, Congcong $L$ and Xiaojun D. Effect of Obesity and Leptin Level on Migraineurs. Med Sci Monit 2015;21 (3270-3274 Doi: 10.12659/msm.894666

17. Nikiforova OS and Delva MY. Neurophysiological features of the nociceptive trigeminal pathway in abdominally obese migraineurs. Wiad Lek 2020;73(4):674-678

18. Ornello R, Ripa P, Pistoia F, Degan D, Tiseo C, Carolei A and Sacco $S$. Migraine and body mass index categories: a systematic review and meta-analysis of observational studies. J Headache Pain 2015;16:27 Doi: 10.1186/s10194-015. 0510-z

19. Razeghi Jahromi S, Ghorbani Z, Martelletti P, Lampl C and Togha M. Association of diet and headache. J Headache Pain 2019;20(1):106 Doi: 10.1186/s10194-019-1057-1

20. Kitamura $\mathrm{E}$, Kanazawa $\mathrm{N}$ and Hamada J. Hyperleptinemia increases the susceptibility of the cortex to generate cortical spreading depression. Cephalalgia 2015;35(4):327-334 Doi: 10.1177/0333102414540813

21. Dang JT, Lee JKH, Kung JY, Switzer NJ, Karmali S and Birch DW. The Effect of Bariatric Surgery on Migraines: a Systematic Review and Meta-analysis. Obes Surg 2020;30(3):1061. 1067 Doi: 10.1007/s1 1695-019-04290-9

22. Miri A, Nasiri M, Zonoori S, Yarahmad F, Dabbagh-Moghadam A, Askari $G, \ldots$ Asadi M. The association between obesity and migraine in a population of Iranian adults: a case-control study. Diabetes Metab Syndr 2018;12(5):733736 Doi: 10.1016/i.dsx.2018.04.020
23. May A and Schulte LH. Chronic migraine: risk factors, mech anisms and treatment. Nat Rev Neurol 2016;12(8):455-464 Doi: 10.1038/nrneurol.2016.93

24. Pavlovic JM, Vieira JR, Lipton RB and Bond DS. Association Between Obesity and Migraine in Women. Curr Pain Headache Rep 2017;21(10):41 Doi: 10.1007/s1 1916-017-0634-8

25. Cho SJ and Chu MK. Risk factors of chronic daily headache or chronic migraine. Curr Pain Headache Rep 2015;19(1):465 Doi: 10.1007/s1 1916-014-0465-9

26. Rossi $\mathrm{HL}$, Lara $\mathrm{O}$ and Recober $\mathrm{A}$. Female sex and obesity increase photophobic behavior in mice. Neuroscience 2016;331:99108 Doi: 10.1016/i.neuroscience.2016.06.022

27. Bond DS, Thomas JG, O'Leary KC, Lipton RB, Peterlin BL, Roth J, ... Wing RR. Objectively measured physical activity in obese women with and without migraine. Cephalalgia 2015;35(10):886-893 Doi: 10.1177/0333102414562970

28. Cervoni C, Bond DS and Seng EK. Behavioral Weight Loss Treatments for Individuals with Migraine and Obesity. Curr Pain Headache Rep 2016;20(2):13 Doi: 10.1007/s1 1916. 016-0540-5

29. Di Vincenzo A, Beghetto M, Vettor R, Tana C, Rossato M, Bond DS and Pagano C. Effects of Surgical and Non-surgical Weight Loss on Migraine Headache: a Systematic Review and Meta-Analysis. Obes Surg 2020;30(6):2173-2185 Doi: 10.1007/s1 1695-020-04429-z

30. Peterlin BL, Sacco S, Bernecker C and Scher Al. Adipokines and Migraine: A Systematic Review. Headache 2016;56(4):622-644 Doi: 10.1111/head. 12788

31. Razeghi Jahromi S, Abolhasani M, Ghorbani Z, Sadre-Jahani S, Alizadeh Z, Talebpour M, . . Togha M. Bariatric Surgery Promising in Migraine Control: a Controlled Trial on Weight Loss and Its Effect on Migraine Headache. Obes Surg 2018;28(1):87-96 Doi: 10.1007/s1 1695-017-2793-4

32. Rainero I, Govone F, Gai A, Vacca A and Rubino E. Is Migraine Primarily a Metaboloendocrine Disorder? Curr Pain Headache Rep 2018;22(5):36 Doi: 10.1007/s1 1916-0180691.7 